\title{
Husbands' participation in prenatal care and breastfeeding self-efficacy in Iranian women: A randomized clinical trial
}

\author{
Soheila Rabiepoor ${ }^{1}$, Alireza Khodaei ${ }^{2}$, Rohollah Valizadeh*3
}

Received: 25 May 2018

Published: 20 Jun 2019

\begin{abstract}
Background: Self-efficacy is an important psychological and motivational factor for continuation of exclusive breastfeeding. This study aimed to examine the effect of husband participation on self-efficacy of breastfeeding in postpartum period.

Methods: This study was a single-blind clinical trial (IRCT2014012115163N2) conducted on 66 pregnant women who referred to health centers of Urmia in 2014. Convenience sampling method was used to select the participants and then they were randomly allocated to control and intervention groups using RANDBETWEEN function in Excel. Participants in the intervention group had 2 training sessions with 4-week interval. They could also use telephone counseling over study time. Those in the control group received the routine pregnancy care, and their breastfeeding self-efficacy was compared 1 month after delivery using Dennis Breastfeeding Self-Efficacy questionnaire. The data were analyzed using SPSS version 20. Chi square, independent and paired samples t test were used.

Results: In this study, the mean score of breastfeeding self-efficacy in the intervention and control groups after intervention was $50.36 \pm 8.65$ and $44.12 \pm 10.41$, respectively, which showed a statistically significant difference $(\mathrm{p}=0.017)$.

Conclusion: According to the results, one of the considerable strategies to enhance self-efficacy of breastfeeding is to involve husbands in prenatal care, which should be considered by the authorities and health professionals, including doctors, nurses, and midwives.
\end{abstract}

Keywords: Breastfeeding, Prenatal care, Self-efficacy

Conflicts of Interest: None declared

Funding: None

*This work has been published under CC BY-NC-SA 1.0 license.

Copyright $\subseteq$ Iran University of Medical Sciences

Cite this article as: Rabiepoor S, Khodaei A, Valizadeh R. Husbands' participation in prenatal care and breastfeeding self-efficacy in Iranian women: A randomized clinical trial. Med J Islam Repub Iran. 2019 (20 Jun);33:58. https://doi.org/10.47176/mjiri.33.58

\section{Introduction}

Self-efficacy is a cognitive and dynamic process that evaluates the ability of people to perform a healthy behavior and it can also predict breastfeeding behaviors that show to what extent the mother is consistent in breastfeeding (1). Dennis believes that mothers with high breastfeeding self-efficacy can maintain exclusive breastfeeding for a longer period (2). Self-efficacy is one of the adjustable variables and an important factor in identifying risk factors for early cessation of breastfeeding, which can be corrected by training (3). If self-efficacy of breastfeeding

\section{Corresponding author: Rohollah Valizadeh, valizadeh.r@tak.iums.ac.ir}

1. Health, Reproductive Health Research Centre, Midwifery Department, Urmia University of Medical Sciences, Urmia, Iran

2. Student Research Committee, Midwifery Department, Urmia University of Medical Sciences, Iran

3. Department of Epidemiology, Student Research Committee, School of Public Health, Iran University of Medical Sciences, Tehran, Iran is higher in mothers, it will be easier for them to continue breastfeeding. Researchers found that mothers who are successful breast feeders are also more successful in raising their children and their children have stronger sense of self-efficacy and acceptance of their mother's role $(4,5)$. Breastfeeding decreases in the first 6 months of lactation, and only $35 \%$ of mothers exclusively breastfeed at fourth month postpartum, which means that the rest of lactating mothers use milk and formula (6). Dennis' study showed that only small number of mothers in developing countries

$\uparrow$ What is "already known” in this topic:

Self-efficacy is one of the adjustable variables and an important factor in identifying risk factors for early cessation of breastfeeding.

\section{$\rightarrow$ What this article adds:}

Involving husbands is one strategy to enhance breastfeeding self-efficacy in prenatal care, which should be considered by the authorities and health professionals, including doctors, nurses and midwives. 
continue to breastfeed 3 months after birth (7).

According to various studies, appropriate strategies and educational interventions can be useful for increasing selfefficacy and individual empowerment (8-10). According to theory of Albert Bandura, self-efficacy is influenced by 4 main information sources: (1) performance accomplishments (eg, previous breastfeeding experience); (2) vicarious experiences (eg, watching other lactating women); (3) verbal persuasion (eg, encouragement from influential people and previous consultations); (4) physiological responses (eg, fatigue, stress, anxiety, sleep disorders) and knowledge $(11,12)$.

It seems that social support and family support, especially support of the spouse, are more important for increasing breastfeeding self-efficacy (13). The results of a study in the UK showed that acceptance of breastfeeding as a norm in the society and social .support of mothers were highly important in improving self-efficacy and continuing breastfeeding (14). Considering the reduced rate of breastfeeding Iran in recent years, appropriate strategies to increase breastfeeding self-efficacy, especially before pregnancy, is needed more than ever (15). Considering the fact that limited studies have been done on fathers' participation in prenatal care on postpartum outcomes, especially breastfeeding, this study aimed to examine the effect of husbands' participation on breastfeeding self-efficacy in postpartum period in women who referred to health center of Urmia in 2014.

\section{Methods}

This was a single-blind clinical trial (IRCT 2014012115163N2; ethic code: 92-01-33-1023) conducted on 66 pregnant women who referred to health center of Urmia in 2014. In this study, inclusion criteria included gestational age between 26-30 weeks, mother's age between 18-35 years, absence of mental illness, uncomplicated pregnancy, not taking psychiatric drugs, wanted pregnancy, and having no contraindication for breastfeeding. Exclusion criteria were dissatisfaction and lack of interest to participate in the study, absence of pregnant women or their partners in training sessions, diagnosis of malformations and fetal or infant death, pregnancy complications at the time of intervention, and having contraindication for breastfeeding. The sample size in this study was calculated based on previous research conducted by Meedya and et $\mathrm{al}^{25}$. Convenience sampling method was used to select the participants and then they were randomly allocated to control and intervention groups using RANDBETWEEN function in Excel.

Sample size was estimated 26 for each group, given

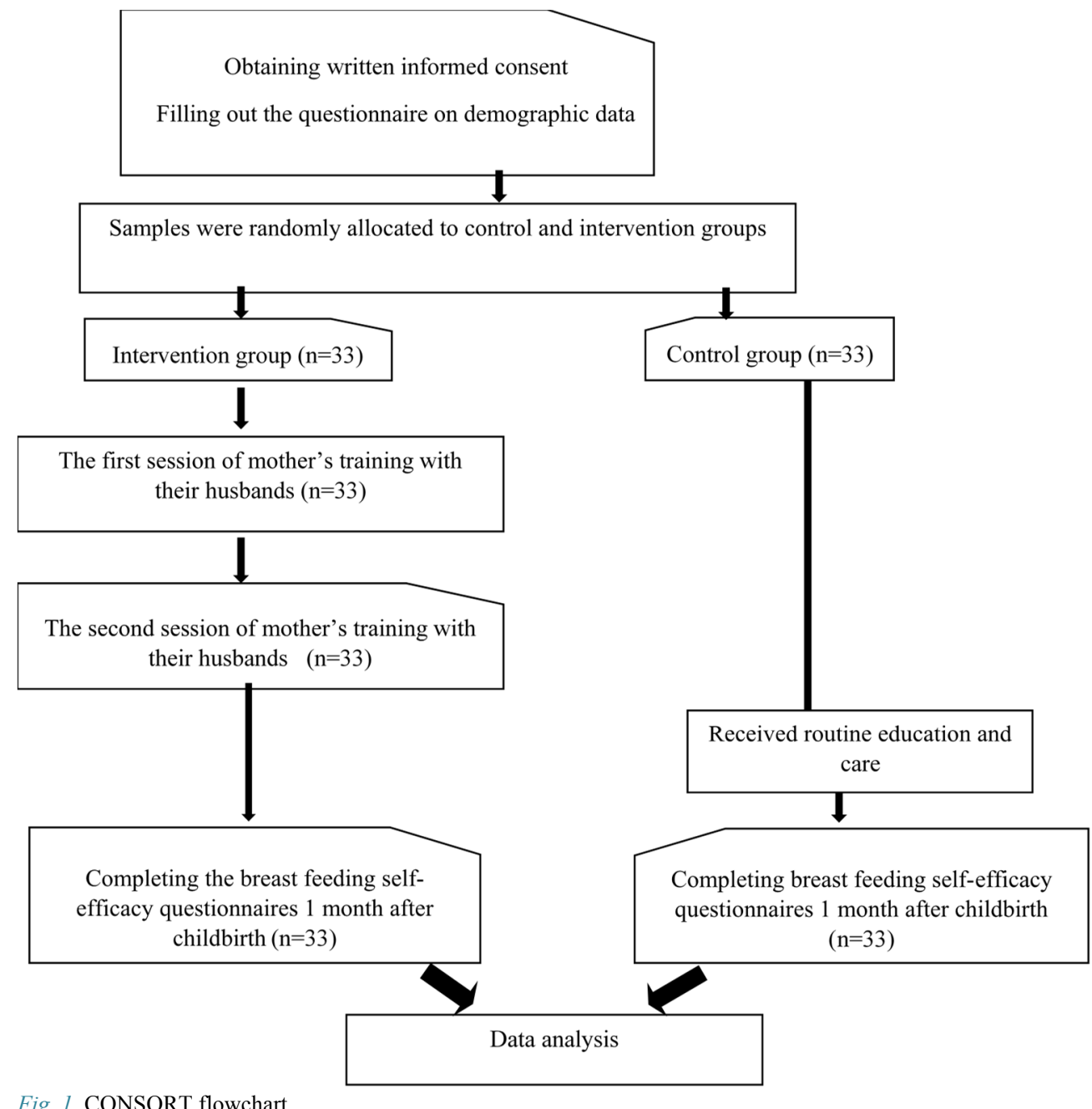


$90 \%$ power and $95 \%$ confidence interval, according to the study by Karbandi et al (18), using the following formula:

$n=\frac{\left(z_{1-\alpha / 2}+z_{1-\beta}\right)^{2}\left({s_{1}}^{2}+s_{2}{ }^{2}\right)^{2}}{\left(\mu_{1}-\mu_{2}\right)^{2}}=\frac{(1.96+1.28)^{2}\left(6.35^{2}+6.79^{2}\right)}{(44.63-50.51)^{2}} \cong 26$

However, considering $20 \%$ attrition rate, 33 pregnant mothers were randomly sampled for the intervention and control groups (Fig. 1).

After explaining the objectives of the study, written informed consent was obtained from all participants. Before the intervention, participants in the intervention group were informed that their husbands' presence was required in the training sessions. For the intervention group, 2 twohour sessions were held with a 4-week interval. The couples had the benefit of telephone counseling during this time and were given a training package that included a training manual about prenatal and postnatal care and lactation, the importance of breastfeeding and its benefits for both the mother and the baby, breastfeeding techniques, notes that mothers should respect, and breastfeeding continuation and its difficulties and how to manage them. The control group received routine education and care. A questionnaire, including self-efficacy of breastfeeding and demographic information, was completed by both groups.

In this study, 2 questionnaires were used to collect data. The first consisted of demographic information of pregnant mothers and their husbands, which was completed at the beginning of the study. The second questionnaire, a standard 14-item questionnaire about breastfeeding selfefficacy in which all questions started with "I Can", was developed by Dennis in 2003.

Breastfeeding self-efficacy questionnaires were completed 1 month after delivery for both groups. Based on a 5-point Likert scale and Bandura theory, the sentences were designed to be affirmative. The range of scores were between 1 , never, or not at all sure, up to 5, always or very confident, for each answer; and the rates of self-efficacy of breastfeeding was between 14 and 70. The highest score indicated the highest breastfeeding efficacy. In other words, if the scores were above the median, the mother had high breastfeeding self-efficacy and, in contrast, the scores below the median indicated that the mother had low breastfeeding self-efficacy. The reliability and validity of the questionnaire was approved in the study of Rahmatnejad et al ${ }^{16}$. Data were analyzed using SPSS-20. Descriptive statistics as mean, standard deviation and frequency, chi-square, independent and paired t test were used to examine the results of this study. To verify the normal distribution of variables, Kolmogorov-Smirnov test was used. Also, significance level was set at $\mathrm{p}<0.05$. The primary outcome measure was postpartum breastfeeding self-efficacy.

\section{Results}

In this study, the mean age in the intervention and control groups was $25.85 \pm 4.64$ and $24.82 \pm 4.29$ years, respectively. The 2 group were not significantly different in demographic characteristics (Table 1).

The demographic characteristics of the husbands showed that $84.8 \%$ of those in the intervention group and $81.8 \%$ in the control group were happy about their children's gender and results showed no significant difference between the intervention and control groups in this regard $(\mathrm{p}=0.740)$. In the intervention group, $45.5 \%(\mathrm{n}=15)$ did not have a high school diploma and $54.5 \%(n=18)$ had diploma and higher education degrees. In the control group, $42.2 \%$ did not have high school diploma and $57.6 \%(n=19)$ had high school diploma and higher degrees. Thus, the 2 groups were not significantly different in education $(\mathrm{p}=0.493)$. Also, $30.3 \%(\mathrm{n}=10)$ of the participants in the intervention group were employed and $69.7 \%$ $(n=23)$ were housewives. In the control group, $45.5 \%$ $(n=15)$ were employed and $54.5 \%(n=18)$ were housewives. The 2 groups did not significantly differ in this regard $(\mathrm{p}=0.588)$.

Table 1. Comparison of the demographic characteristics of mothers in the intervention and control groups

\begin{tabular}{|c|c|c|c|c|c|}
\hline \multirow[t]{2}{*}{ Variables } & & \multicolumn{2}{|c|}{ Intervention group } & \multicolumn{2}{|c|}{ Control group } \\
\hline & & Frequency & Percent & Frequency & Percent \\
\hline \multirow[t]{3}{*}{ Age of mothers (years) } & $18-22$ & 8 & 24.2 & 12 & 36.4 \\
\hline & $23-27$ & 14 & 42.4 & 10 & 30.3 \\
\hline & $28-34$ & 11 & 33.3 & 11 & 33.3 \\
\hline \multirow{3}{*}{ Number of pregnancy } & 1 & 10 & 30.3 & 11 & 33.3 \\
\hline & 2 & 16 & 48.5 & 19 & 57.6 \\
\hline & 3 and more & 7 & 21.2 & 3 & 9.1 \\
\hline \multirow{2}{*}{ Number of delivery } & 0 & 10 & 30.3 & 11 & 33.3 \\
\hline & 1 and more & 23 & 69.7 & 22 & 66.7 \\
\hline \multirow[t]{2}{*}{ Number of live child } & 0 & 11 & 33.3 & 12 & 36.4 \\
\hline & 1 and more & 22 & 66.7 & 21 & 63.6 \\
\hline \multirow[t]{2}{*}{ Mother's education level } & Below the diploma & 15 & 45.5 & 14 & 42.4 \\
\hline & Diploma and above & 18 & 54.5 & 19 & 57.6 \\
\hline \multirow[t]{2}{*}{ Asked the child sex } & No & 27 & 81.8 & 25 & 75.8 \\
\hline & Yes & 6 & 18.2 & 8 & 24.2 \\
\hline \multirow[t]{3}{*}{ Economic status } & Favorable & 8 & 24.2 & 10 & 30.3 \\
\hline & Somewhat favorable & 18 & 54.5 & 17 & 51.5 \\
\hline & No favorable & 7 & 21.2 & 6 & 18.2 \\
\hline \multirow{2}{*}{ Occupation of mother } & Employed & 10 & 30.3 & 15 & 45.5 \\
\hline & Housewife & 23 & 69.7 & 18 & 54.5 \\
\hline \multirow[t]{3}{*}{ Housing } & Tenant & 8 & 24.2 & 13 & 39.4 \\
\hline & Owner & 18 & 54.5 & 15 & 45.5 \\
\hline & Live with family & 7 & 21.2 & 5 & 15.2 \\
\hline
\end{tabular}


Independent $\mathrm{t}$ test was performed to compare breastfeeding self-efficacy of the 2 groups. The results shown in Table 2 suggest that breastfeeding self-efficacy was significantly different in the 2 groups after intervention.

Table 3 demonstrates a significant relationship between demographic characteristics and breastfeeding selfefficacy of mothers in the intervention group. Also, the number of pregnancy, number of children, and education had a statistically significant relationship with breastfeeding self-efficacy in the intervention group. However, there was no significant relationship among mother's age, parity, being asked about the gender of the child, economic status, and mother's employment with breastfeeding selfefficacy.

\section{Discussion}

The results of this study showed that the rate of breastfeeding self-efficacy was more in the intervention group than in the control group, and this difference was statistically significant. Moreover, the results indicated that familial support in breastfeeding is an important factor in the self-efficacy of mothers, which was consistent with the third step of the theory of breastfeeding self-efficacy, which points out that self-efficacy of the mother improves when she receives verbal encouragement from people who are important to her (17).

The results of this study were consistent with studies of Pisacane et al (18), Earle et al (19), Cohen et al (20), and Kenosi et al (21), that showed the role of partners in the success of breastfeeding. However, the results of this study did not match with those of Henderson et al (22) that revealed breastfeeding self-efficacy score in the intervention group was less than the control group. The reason for this difference may be due to the time of the training period in the first 24 hours after delivery. On the other hand, Freed et al (23) and Giuliani et al (24) showed that lack of awareness of husbands of pregnant women about the benefits of breastfeeding can affect the rate of breastfeeding and its continuation. Therefore, to raise fathers' awareness and support, they should be with their pregnant wives when they are receiving breastfeeding training (25).

The study of Zhu et al (26) have shown that perceived social support by mothers and attention from important people (eg, husband, mother, friends), in addition to previous experience of previous breastfeeding, could be related to breastfeeding self-efficacy in Chinese women. On the other hand, Otsuka et al (27) stated that increased breastfeeding self-efficacy in postpartum period may reduce mother's perceived insufficient milk, early cessation of breastfeeding, and giving milk supplements to the child during breastfeeding. Therefore, educational intervention for pregnant women's spouses can include emotional support and increase self-efficacy of lactation and health care (18). The results showed that breastfeeding efficacy had a statistically significant association with age, economic status, and employment in the intervention group. The results of the present study were consistent with those of Azhari et al (15), Bastani et al (28), Blyth et al (29), and Denis et al (33). However, they were different from those of Mercer et al (31) and Varaei et al (32). The difference in the results may be due to cultural and demographical differences of the samples.

This study revealed a statistically significant and direct correlation between the efficacy of breastfeeding and mother's education level. The results were consistent with those of McQueen et al (33) and were inconsistent with the study of Otsuka (27). The difference in results may be due to both the community, education level of mothers, and use of mass media such as television.

Table 2. Comparison of breastfeeding self-efficacy scores between the intervention and control groups after their husbands participation in prenatal care

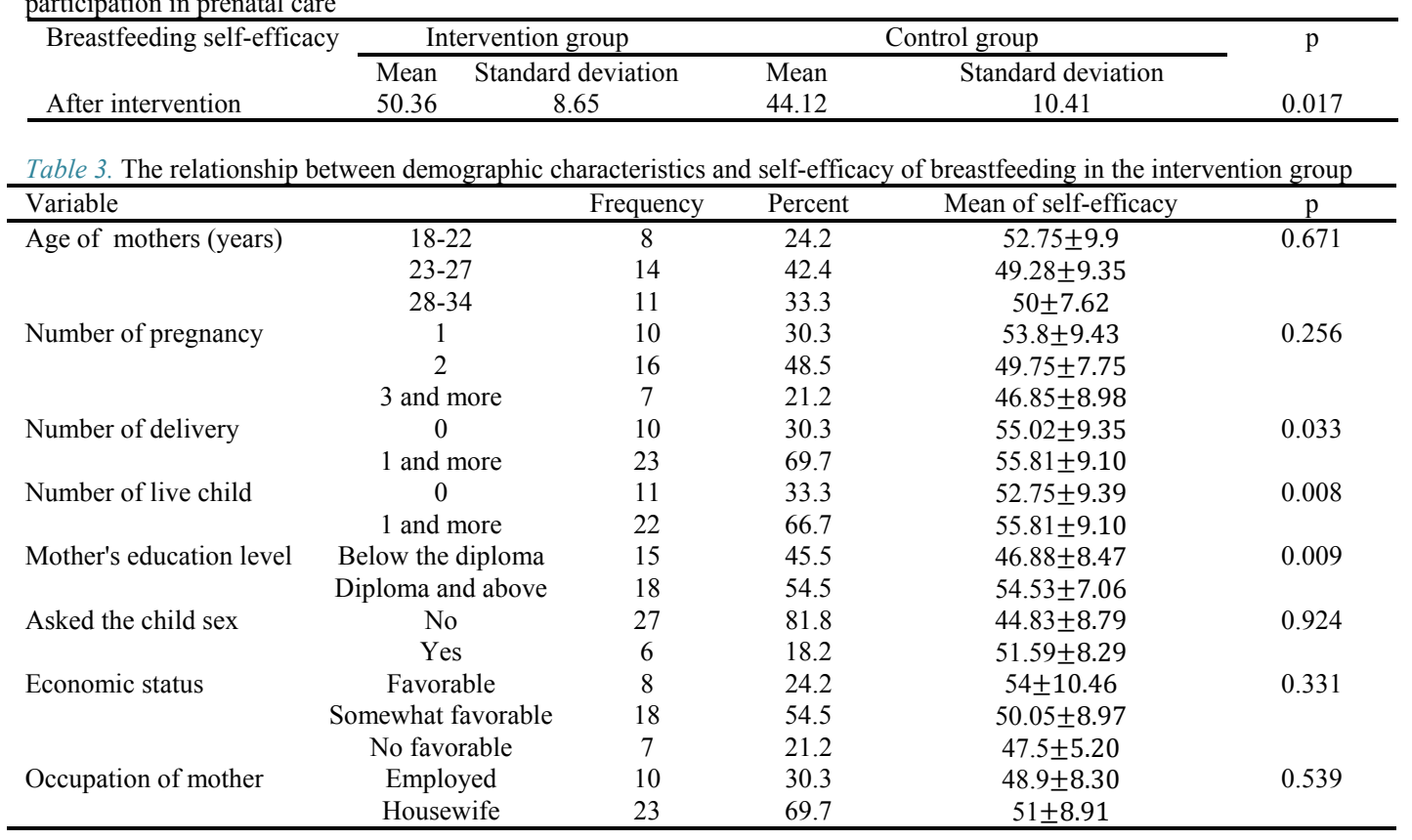




\section{Limitations}

One of the potential limitations of this study was the small number of studies conducted on the effect of husbands' participation in breastfeeding. It is recommended to conduct further studies to obtain additional information on breastfeeding self-efficacy.

\section{Conclusion}

Breastfeeding self-efficacy is a potentially modifiable factor that can predict breastfeeding duration and level. One of the strategies to enhance self-efficacy of breast feeding is participation of husbands during pregnancy and lactation, which should be considered by the authorities and health professionals, including doctors, nurses, and midwives. Breastfeeding self-efficacy is an appropriate theoretical framework to guide interventions that can increase the duration of breastfeeding and improve children's health. In addition, it is a valid and reliable tool for identifying at-risk women.

\section{Acknowledgments}

This was a clinical trial (IRCT2014012115163N2 code) registered at the center of registered clinical trials and was derived from a master thesis in nursing. The authors would like to acknowledge and thank the deputy of research at School of Nursing and Midwifery of Urmia, all expecting mothers and their husbands, and health care workers of Shohada, Vakilabad, Islamabad, and number 6 health care centers for their invaluable cooperation and support.

\section{Conflict of Interests}

The authors declare that they have no competing interests.

\section{References}

1. Gijsbers B, Mesters I, Knottnerus JA, Van Schayck CP. Factors associated with the duration of exclusive breast-feeding in asthmatic families. Health Educ Res. 2008;23(1):158-69.

2. Blyth R, Creedy DK, Dennis CL, Moyle W, Pratt J, De Vries SM. Effect of maternal confidence on breastfeeding duration: An application of breastfeeding self-efficacy theory. Birth. 2002;29(4):278-84.

3. Khorsandi M, Ghofranipour F, Faghihzadeh S, Hidarnia A, Akbarzadeh Bagheban A, Aguilar-Vafaie ME. Iranian version of childbirth self-efficacy inventory. J Clin Nurs. 2008;17(21):2846-55.

4. McCarter-Spaulding DE, Kearney MH. Parenting Self-Efficacy and Perception of Insufficient Breast Milk. J Obstet Gynecol Neonatal Nurs. 2001;30(5):515-22.

5. Edmond KM, Zandoh C, Quigley MA, Amenga-Etego S, OwusuAgyei S, Kirkwood BR. Delayed breastfeeding initiation increases risk of neonatal mortality. Pediatrics. 2006;117(3):e380-e6.

6. Barber CM, Abernathy T, Steinmetz B, Charlebois J. Using a breastfeeding prevalence survey to identify a population for targeted programs. Can J Public Health. 1996;88(4):242-5.

7. Dennis CL. Breastfeeding Initiation and Duration: A 1990-2000 Literature Review. J Obstet Gynecol Neonatal Nurs. 2002;31(1):1232.

8. Bandura A. Self-efficacy: toward a unifying theory of behavioral change. Psychol Rev. 1977;84(2):191.

9. Hannula L, Kaunonen M, Tarkka MT. A systematic review of professional support interventions for breastfeeding. J Clin Nurs. 2008;17(9):1132-43.

10. Weimers L, Svensson K, Dumas L, Navér L, Wahlberg V. Hands-on approach during breastfeeding support in a neonatal intensive care unit: a qualitative study of Swedish mothers' experiences. Int Breastfeed J. 2006;1(1):20.

11. Rabiepoor S, Valizadeh R, Barjasteh S. Study of Menstrual Attitudes and Knowledge among Postmenarcheal Students, in Urmia, North West of Iran. Int J Pediatr 2017; 5(5): 4991-5001.

12. Dennis CL. Theoretical Underpinings of Breastfeeding Confidence: A Self-Efficacy Framework. J Hum Lact. 1999;15:195-202.

13. DiGirolamo A, Thompson N, Martorell R, Fein S, Grummer-Strawn L. Intention or experience? Predictors of continued breastfeeding. Health Educa Behav. 2005;32(2):208-26.

14. Brown A, Raynor P, Lee M. Young mothers who choose to breast feed: the importance of being part of a supportive breast-feeding community. Midwifery. 2011;27(1):53-9.

15. Azhari S, Baghani R, Akhlaghi F, Ebrahimzadeh S, Salehi Federdi J. Comparing the effects of hands-on and hands-off breastfeeding methods on self-efficacy in primiparous mothers. J Sabzevar Uni Med Sci. 2011;17(4):248-55.

16. Rahmatnejad L, Bastani F. An investigation of Breast Feeding Self Efficacy and its Relationship with exclusive breast feeding. Alborz Uni Med J. 2012;1(1):31-6.

17. Wells KJ, Thompson NJ, Kloeblen-Tarver AS. Development and psychometric testing of the prenatal breast-feeding self-efficacy scale. Am J Health Behav. 2006;30(2):177-87.

18. Karbandi S, Hosseini SM, Masoudi R, Mamori GH. The effect of relaxation training on breastfeeding self-efficacy of mothers with preterm infants: A randomized clinical trial. J Clin Nurs Midwifery. 2014; 3(2): 37-45.

19. Earle S. Why some women do not breast feed: bottle feeding and fathers' role. Midwifery. 2000;16(4):323-30.

20. Cohen R, Lange L, Slusser W. A description of a male-focused breastfeeding promotion corporate lactation program. J Hum Lact. 2002;18(1):61-5.

21. Kenosi M, Hawkes C, Dempsey E, Ryan C. Are fathers underused advocates for breastfeeding? Irish Med J. 2012 May;105(5):157-8.

22. Henderson A, Stamp G, Pincombe J. Postpartum positioning and attachment education for increasing breastfeeding: a randomized trial. Birth. 2001;28(4):236-42.

23. Giugliani E, Bronner Y, Caiaffa W, Vogelhut J, Witter F, Perman J. Are fathers prepared to encourage their partners to breast feed? A study about fathers' knowledge of breast feeding. Acta Paediatr. 1994;83(11):1127-31.

24. Freed GL, Fraley JK, Schanler RJ. Attitudes of expectant fathers regarding breast-feeding. Pediatrics. 1992;90(2):224-7.

25. Meedya S, Fahy K, Yoxall J, Parratt J. Increasing breastfeeding rates to six months among nulliparous women: a quasi-experimental study. Midwifery. 2014 Mar;30(3):e137-44.

26. Zhu J, Chan WCS, Zhou X, Ye B, He H-G. Predictors of breast feeding self-efficacy among Chinese mothers: A cross-sectional questionnaire survey. Midwifery. 2014;30(6):705-11.

27. Otsuka K, Dennis CL, Tatsuoka H, Jimba M. The relationship between breastfeeding self-efficacy and perceived insufficient milk among Japanese mothers. J Obstet Gynecol Neonatal Nurs. 2008;37(5):546-55.

28. Bastani F, Rahmatnejad L, Jahdi F, Haghani H. Breastfeeding self efficacy and perceived stress in primiparous mothers. Iran J Nurs. 2008;21(54):9-24.

29. Blyth RJ, Creedy DK, Dennis C-L, Moyle W, Pratt J, De Vries SM, et al. Breastfeeding duration in an Australian population: the influence of modifiable antenatal factors. J Hum Lact. 2004;20(1):308.

30. Dennis CLE. Identifying predictors of breastfeeding self-efficacy in the immediate postpartum period. Res Nurs Health. 2006;29(4):25668.

31. Mercer RT. A theoretical framework for studying factors that impact on the maternal role. Nurs Res. 1981;30(2):73-7.

32. Varaei S, Mehrdad N, Bahrani N. The Relationship between Selfefficacy and Breastfeeding, Tehran, Iran. Hayat. 2009;15(3):31-8.

33. McQueen KA, Dennis CL, Stremler R, Norman CD. A pilot randomized controlled trial of a breastfeeding self-efficacy intervention with primiparous mothers. J Obstet Gynecol Neonatal Nurs. 2011;40(1):35-46. 